Multiplicités des approches en anglais de spécialité

\title{
Muriel Grosbois, Didactique des langues et technologies : de l'EAO aux réseaux sociaux
}

Paris : Presses de l'Université Paris-Sorbonne, 2012

\section{Noëlla Gaigeot}

\section{(2) OpenEdition}

\section{Journals}

Édition électronique

URL : http://journals.openedition.org/asp/3272

DOI : $10.4000 /$ asp.3272

ISSN : 2108-6354

\section{Éditeur}

Groupe d'étude et de recherche en anglais de spécialité

Édition imprimée

Date de publication : 1 mars 2013

Pagination : 165-168

ISSN : 1246-8185

\section{Référence électronique}

Noëlla Gaigeot, « Muriel Grosbois, Didactique des langues et technologies : de l'EAO aux réseaux sociaux », ASp [En ligne], 63 | 2013, mis en ligne le 01 mars 2013, consulté le 02 novembre 2020. URL : http:// journals.openedition.org/asp/3272 ; DOI : https://doi.org/10.4000/asp.3272

Ce document a été généré automatiquement le 2 novembre 2020

Tous droits réservés 
Muriel Grosbois, Didactique des langues et technologies : de l'EAO aux réseaux sociaux

Paris : Presses de l'Université Paris-Sorbonne, 2012

Noëlla Gaigeot

\section{RÉFÉRENCE}

Grosbois, Muriel. 2012. Didactique des langues et technologies : de l'EAO aux réseaux sociaux. Paris : Presses de l'Université Paris-Sorbonne, 177 p. ISBN 978-2-84050-848-9. 
Dans son ouvrage, intitulé Didactique des langues et technologies: de l'EAO aux réseaux sociaux, l'objectif de Muriel Grosbois, enseignant-chercheur à l'Université ParisSorbonne est de proposer un panorama de l'usage des technologies pour l'enseignement-apprentissage des langues qui éclaire les pratiques actuelles et en émergence, avec le recul réflexif qu'une telle approche permet. Elle plaide ainsi pour un usage raisonné des technologies au service de la formation. Des exemples de projets en cours en anglais, en allemand et en français langue étrangère (FLE) viennent illustrer le propos. Cet ouvrage prend appui sur un champ de recherche qui s'articule autour de la linguistique et de la didactique; il combine théorie et pratique, expérimentations et propositions pédagogiques. Il est structuré en huit chapitres, selon une trame chronologique, avec un découpage en quatre périodes : les années 1960 à 1990, les années 1990 à 2000, les années 2000 et enfin les courants les plus récents.

Dans son premier chapitre intitulé «Un peu d'histoire du domaine - Les années 60 à 90 », M. Grosbois porte un regard historique sur l'usage des technologies pour l'enseignement-apprentissage des langues et analyse les temps forts définis autour de la machine elle-même, «l'ordinateur tuteur», «l'ordinateur apprenant», «l'ordinateur outil», et autour de la conception de l'apprentissage, «l'apprentissage par l'instruction », " l'apprentissage par l'action », « l'apprentissage par consultation ». Elle rappelle que, dans les années 1960, quand on parle de technologie (éducative), celle-ci se place dans le cadre du béhaviorisme et de l'enseignement programmé sans pour autant qu'il soit nécessaire d'avoir recours à des machines. Les objets qui aident à l'enseignement programmé sont des livres programmés («programmation linéaire » selon Skinner [1961], ou "ramifiée » selon Crowder [1960]) et la technologie n'est qu'une simple série de techniques et d'outils de construction de cours. L'apprenant réussit ou non à avoir le comportement attendu de lui, comportement observé dans un cadre théorique de stimulus, réponse, renforcement positif ou négatif, et il passe à l'étape suivante seulement si l'étape précédente est maîtrisée.

3 L'ère de l'enseignement assisté par ordinateur (EAO) débute entre la fin des années 1960 et le début des années 1970 et il s'agit alors de produire des cours. Tutoriels, exerciseurs et tests, vite regroupés sous le terme de didacticiels, font leur apparition. L'apprenant évolue dans le cadre d'un scénario, ou il se voit proposer des exercices répétitifs d'application-fixation, ou encore il se teste via la machine-tuteur. Dans son deuxième chapitre «Les années 60 à 90 - Lignes de force ", M. Grosbois rend compte des changements qui émergent. L'accent est mis sur la liberté et l'initiative de l'apprenant. Les nouvelles approches se réclament du constructivisme et de la psychologie cognitive qui s'accordent sur une conception de l'apprentissage reposant sur un processus dynamique de construction des connaissances. La machine devient 
alors «ordinateur apprenant» puis « ordinateur outil». L'apprenant doit prendre l'initiative, agir et observer le résultat de ses actions. Cette période correspond à "l'apprentissage par l'action » et se différencie de "l'apprentissage par l'instruction » qui mettait auparavant l'apprenant dans une situation de reproduction d'un modèle explicité via un médium. Tout au long de ces trente années commencent donc à se dégager certaines « lignes de force ». La question de savoir comment apprendre se pose de façon plus prégnante : l'apprentissage doit-il être libre ? Doit-il être guidé ? Doit-il confronter l'apprenant à des systèmes-auteurs ouverts ou fermés? Faut-il une interactivité ? Cette période pose l'ordinateur comme un moyen d'individualiser le travail de l'apprenant, de le faire sortir du groupe et de déplacer la relation à l'enseignant.

4 Dans le chapitre 3, M. Grosbois présente les avancées de la décennie 1990: le multimédia, l'hypermédia, les technologies de l'information et de la communication (TIC) et Internet. C'est l'époque où la communication prend le pas sur l'information. Des actions pédagogiques liées à l'usage d'Internet, notamment la recherche d'informations, la cyberquête, la cyberenquête ou l'enquête virtuelle se développent. L'apprenant explore, extrait, consulte, conçoit ou transforme. Il développe son autonomie dans le cadre de dispositifs visant l'autoformation guidée, disposant de ressources incluant les TIC. Il se voit proposer des plages de travail individuel et un accompagnement sous forme de tutorat. La notion d'auto-apprentissage est posée dès le début des années 1990.

5 Désormais liés aux dispositifs pédagogiques ${ }^{1}$ mis en place, les questionnements sur l'autonomie ${ }^{2}$ des apprenants font l'objet du chapitre 4. L'autonomie est-elle un prérequis ou un objectif à atteindre? Les réponses varient en fonction des positionnements didactiques retenus. Faut-il apprendre à travailler en autoformation avant de pouvoir expérimenter l'autonomie? Faut-il s'appuyer sur différents profils d'apprentissage et aider les apprenants à prendre conscience de leur style cognitif ? Se pose aussi la question de la nécessité d'administrer ou non un test diagnostic à l'entrée du parcours de formation, d'élaborer ou non un contrat avec l'apprenant. La mise à disposition des matériaux-ressources est mise en question également dans ces différents dispositifs ainsi que le type d'encadrement mis en place. «On constate que l'approche didactique s'inscrit de plus en plus dans le courant constructiviste et socioconstructiviste, selon lequel l'apprenant construit son savoir avec l'aide de son entourage » (page 67).

Dans les chapitres 5 et 6 (les années 2000), M. Grosbois explore le lien entre l'évolution des supports et leur impact sur les contenus et les approches didactiques, ainsi que leur mise en œuvre, et elle met en regard didactique et usage des technologies. La palette des moyens s'est élargie et le recours à des outils plus spécifiques de la formation à distance trouve un écho dans les contenus et les approches didactiques; l'auteur cite notamment la visioconférence, le courrier électronique, les listes de diffusion, les forums de discussion, les outils de clavardage, les blogs, les wikis, la baladodiffusion, les systèmes logiciels de gestion de formation et d'enseignement. La tendance est à l'approche (inter)actionnelle. La théorie de l'action se combine à la dimension sociale où agir et communiquer se conjuguent dans un contexte d'interaction sociale. Dans cette optique, l'approche par tâches (macro-tâches et micro-tâches) permet à l'apprenant de répondre à des objectifs tout en facilitant l'apprentissage. Le recours aux technologies contribue à la mise en place de cette approche par tâches et offre aux 
apprenants un rôle d'acteurs sociaux conduits à interagir afin de résoudre une situation-problème. Les situations d'apprentissage proposées depuis les années 2000 tendent à articuler l'individuel et le collectif par le biais de tâches effectuées en présentiel et à distance, grâce à l'usage des technologies. L'approche plurilingue et multiculturelle est, elle aussi, facilitée par les développements technologiques. Désormais, une concordance entre les courants didactiques et technologiques devient évidente.

Quatre projets émanant de recherches doctorales viennent illustrer, dans le septième chapitre, les idées-forces dégagées tout au long de l'ouvrage de M. Grosbois : à l'IUFM de Paris, la communication médiée (ou médiatisée) par ordinateur entre locuteurs natifs et non natifs, sous-tendue par un projet de création d'une ressource numérique, déclenche un processus d'apprentissage de l'anglais ${ }^{3}$; l'usage des concordanciers pour le développement de la production écrite en L2 de jeunes apprenants d'allemand, analysé par Eva Schaeffer-Lacroix ${ }^{4}$; les deux autres exemples relèvent de la formation à distance et s'adressent à un public universitaire dans le cadre d'une formation de FLE 5 . Ces types d'approche s'inscrivent dans une démarche de recherche-action, qui invite «à questionner l'artefact qu'est l'outil technologique » (page 124).

Dans le dernier chapitre consacré aux courants les plus récents, eu égard non pas nécessairement à leur date d'émergence, mais à leur évolution, à leur mise en œuvre pédagogique et à leur place dans le domaine de la recherche, M. Grosbois évoque cette dimension collective qui est particulièrement caractéristique des courants les plus récents avec l'émergence des jeux sérieux ("serious games ») et l'évolution des réseaux sociaux. La question soulevée est celle du potentiel réel pour l'apprentissage des langues. Elle est posée aussi bien dans le cadre des jeux vidéo (jeux de simulation, jeux en ligne multijoueurs) ou des mondes virtuels ou synthétiques, que du recours au tableau blanc interactif, aux tablettes numériques, au web2 (qui a donné la possibilité aux réseaux sociaux de se construire).

L'ouvrage offre, dans une langue accessible et précise, un panorama historique de l'usage des technologies et de son action sur la didactique des langues; il fourmille de références utiles. Il s'adresse à tous les étudiants (de licence, master et doctorat) intéressés par la didactique des langues et les technologies ainsi qu'aux professionnels de la formation, aux enseignants de langues et aux chercheurs du domaine. L'intérêt de cette publication, outre le rappel historique, est de poser les limites de ces développements technologiques tout en recensant leur potentiel et leurs dérives possibles. Même si la lecture de cette étude peut laisser une impression de catalogue alternant des chapitres de longueur inégale (par exemple, le premier chapitre compte quinze pages, le quatrième six et le septième trente-deux), elle permet cependant de se poser des questions essentielles sur le plan de la didactique des langues, environnement multiple et dense, et de replacer cette recherche-action dans le cadre d'autres démarches de recherche. En plantant tout au long de son ouvrage jalons historiques et méthodologiques, M. Grosbois contribue à élaborer une réflexion critique sur l'interaction entre la didactique des langues et la liberté dévolue aux espaces de recherche :

Mon positionnement, on l'aura compris, consiste à envisager la recherche en didactique comme un questionnement théorique dont les réponses conduisent à un enrichissement à la fois de la théorie et de la pratique, c'est-à-dire à combiner l'intérêt scientifique et social de la recherche. (page 153) 


\section{BIBLIOGRAPHIE}

Crowder, N. A. 1960. « Automatic tutoring by intrinsic programming ». In Lumsdaine A.A. \& R. Glaser (dir.), Teaching machines and programmed learning: A source book. Washington, D.C. : National Education Association, Department of Audiovisual Instruction, 286-2986.

Skinner, B. F. 1961. « Teaching machines ». Scientific American 205/2, 90-102.

\section{NOTES}

1. Par exemple, le CNEAO (Centre national d'enseignement assisté par ordinateur, Universités Paris 7 et Paris 6), le SAPAG (Service d'apprentissage et de perfectionnement et autoformation guidée à l'Université de Bordeaux), le CNAM (Conservatoire national des arts et métiers à Paris), le CRAPEL (Centre de recherches et d'applications pédagogiques en langues à l'Université de Nancy).

2. M. Grosbois cite Holec, H. 1979, Autonomie et apprentissage des langues étrangères, Strasbourg, Conseil de la coopération culturelle du Conseil de l'Europe.

3. Grosbois, M. 2006. "Projet collectif de création d'une ressource numérique comme levier d'apprentissage de l'anglais ». Thèse de doctorat, Université de la Sorbonne Nouvelle.

4. Schaeffer-Lacroix, E. 2009. "Corpus numériques et production écrite en langue étrangère». Thèse de doctorat, Université de la Sorbonne Nouvelle.

5. Chachkine, E. (2011) analyse la formation à distance sur plate-forme: "Quels scénarios pédagogiques pour un dispositif multimédia d'apprentissage à distance socioconstructiviste et de conception énonciativiste en français langue étrangère ?». Thèse de doctorat, Université d'AixMarseille; et Yun, H. (2009) travaille sur l'usage du clavardage par des apprenants de FLE: «Échanges à distance entre apprenants de FLE - Étude des interactions synchrones en contexte académique ». Thèse de doctorat, Université Sorbonne nouvelle.

6. NDLR : Les coquilles dans la référence ont été corrigées.

\section{AUTEURS}

\section{NOËLLA GAIGEOT}

Université du Maine 